\title{
Fluorescence visualization efficacy for detecting oral lesions more prone to be dysplastic and potentially malignant disorders: a pilot study
}

\author{
Luciana Estevam Simonato ${ }^{\mathrm{a}}$, Saygo Tomo ${ }^{\mathrm{b}, *}$, Glauco Issamu Miyaharab \\ Ricardo Scarparo Navarro ${ }^{a}$, Antonio Guillermo Jose Balbin Villaverde ${ }^{a}$ \\ a Biomedical Engineering Institute, Camilo Castelo Branco University- UNICASTELO, São José dos Campos, SP, Brazil \\ b Oral Oncology Center, Araçatuba Dental School, UNESP_Univ Estadual Paulista, Araçatuba, Brazil
}

\section{A R T I C L E I N F O}

\section{Article history:}

Received 28 July 2016

Received in revised form 19 September 2016

Accepted 27 October 2016

Available online 29 October 2016

\section{Keywords:}

Optical imaging

Diagnosis

Oral

Early diagnosis

Mouth neoplasms

\begin{abstract}
A B S T R A C T
Background: To analyze the efficacy of fluorescence visualization in early detection of oral early neoplastic processes when used by an inexperienced and an adept oral examiner.

Methods: A dental student and an expert professional in oral diagnosis examined 15 randomly selected patients using conventional oral examination and fluorescence visualization searching for early signs of epithelial dysplasia and oral potentially malignant disorders. Patients detected with oral lesions in any of examinations were submitted to incisional biopsy for definitive diagnosis.

Results: Taking histopathologic analysis as Gold Standard, sensitivity, specificity, positive and negative predictive values and accuracy of inexperienced examiner for detecting both epithelium dysplasia and oral potentially malignant disorders increased when using fluorescence visualization compared to conventional white light examination, matching the same values found for expert professional in oral diagnosis. Furthermore, it was possible to observe discrete increase in the efficacy of expert professional's examination when using fluorescence visualization.

Conclusion: This study indicates fluorescence visualization is capable of improve inexperienced professionals' efficacy for early detecting oral lesions more prone to be dysplastic and oral potentially malignant disorders.
\end{abstract}

(C) 2016 Elsevier B.V. All rights reserved.

\section{Introduction}

With the increase in oral squamous cell carcinoma (OSCC) incidence around the world and its association with poor chances of cure and 5-year survival, which might be due to high rates in delayed diagnosis, the development of methods which might help to detect early signs of neoplastic transformation have become mandatory $[1,2]$. Due to the difficulty in early diagnosis of oral cancer, it is important to search and discuss aids which might help to improve clinical examination. Therefore, since 1924, when Policard [3] began to study fluorescence applied to oncology, intense efforts have been given from biomedical engineering and oral medicine researchers regarding the use of fluorescence techniques for early

\footnotetext{
* Corresponding author at: Oral Oncology Center, UNESP-Univ Estadual Paulista, Araçatuba, São Paulo, Brazil, José Bonifácio St, 1193, Araçatuba, São Paulo, 16015050, Brazil.

E-mail address: saygotomo@hotmail.com (S. Tomo).
}

detecting oral lesions more prone to be dysplastic and oral potentially malignant disorders (OPMD) [4,5].

Fluorescence visualization method was developed aiming to provide oral clinicians an aid for evidencing early signs of neoplastic processes [6]. This method consists in emitting fluorescent violet light $(400 \mathrm{~nm}$ ) over oral mucosa through LEDs systems, and observing its response to this stimulus. Normal oral mucosa will disperse and absorb a fraction of the energy applied over it and reflect the greatest part on a larger wavelength $(450-500 \mathrm{~nm})$, presenting as an apple-green fluorescence when observed through optical filters which blocks the incident light [6-8]. Once neoplastic processes are characterized by molecular and morphologic changes, this tissue will disperse and absorb the greatest part of emitted energy, therefore, reflected wavelengths are out of human eye visible spectrum region and neoplastic tissue will present as dark site in oral mucosa (fluorescence loss) [6,9].

When the first prototype of fluorescence visualization handheld device was developed, Lane et al. (2006) [8], found that among 44 patients, sensitivity and specificity values for this device were, 
respectively, $98 \%$ and $100 \%$ for discriminating normal mucosa from severe dysplasia/carcinoma in situ. Later in 2009, Huff et al. [10], using a commercialized fluorescence visualization device, found that this method was capable of increase the detection rate of oral abnormalities, most of which were OPMD. Hanken et al. (2013) [11], demonstrated values of sensitivity and specificity of $97 \%$ and $41 \%$, respectively, using the same device as Huff et al., concluding the method might help on the experienced examiners' clinical examination. Sweeny et al. [12], in 2011, reported fluorescence visualization with high sensitivity (81\%) and specificity (50\%) values, nevertheless, oral examination under conventional white-light demonstrated higher sensitivity (98\%) and similar specificity (50\%), not supporting the efficacy of fluorescence visualization. Therefore, contradictory results highlight the need for further studies assessing the efficacy of this method for early detecting oral lesions prone to be dysplastic, OPMD and OSCC.

Due to variable results in literature regarding the efficacy of fluorescence visualization in detecting oral lesions potentially dysplastic, OPMD and OSCC, and the lack of studies evaluating the potential of this method for enhancing unskilled professionals capacity in detecting these lesions, the aim of this pilot study was to evaluate fluorescence visualization for early detecting oral lesions potentially dysplastic and OPMD in general population scanning, when used by an unskilled and an adept examiner in oral diagnosis, and compare to the conventional white-light clinical examination performed by both examiners.

\section{Materials and methods}

\subsection{Ethical approval}

This study was performed after approval by the ethics committee for research of the Camilo Castelo Branco University (UNICASTELO) through the protocol no. 43602515.5.0000.5494.

\subsection{Samples}

15 patients were randomly selected from the screening clinic of the UNICASTELO dentistry course and invited to participate in the study, accepting through signing informed consent term. Of these patients, 11 (73.33\%) were men, four (26.66\%) were women, and the average age was 52.13 years. Two (13.33\%) patients were considered smokers, and six (40\%) alcoholic.

Adept professional in oral diagnosis was represented by a Doctor of Dental Surgery (DDS) and Master of Science (MSc) of oral medicine area, while unskilled examiner was represented by a dental student, who had already undergone clinical training for oral examination. Examiners also accepted to participate in the study by signing informed consent term. Both examiners performed oral conventional and fluorescence visualization examination on the same 15 patients, therefore, each patient underwent four oral examinations.

\subsection{Oral conventional clinical examination (white-light examination)}

For oral conventional clinical examination, both examiners were calibrated through training lecture performed by a further professional adept in oral diagnosis. Therefore, this examination was systematically performed, observing and palpating every oral structure, with the patient seated in a dental chair. Then, the examiners reported the positivity or negativity of oral lesion on patients' individual records.

\subsection{Fluorescence visualization examination}

Following the oral conventional clinical examination, both examiners performed the examination using the handheld device EVINCE $^{\circledR}$ (MMOptics, São Carlos, SP, Brazil), which emits light in $400 \mathrm{~nm}$ wavelength through LEDs system, and allows the observation of tissue autofluorescence through optical filter linked to the device.

This examination was performed in dark room, aiming to avoid the interference of white-light on the observation of tissue fluorescence. The examiners were equally calibrated for using the device and performed the examination systematically, observing the fluorescence of all oral structures. When noticing a well demarcated area of fluorescence loss (dark region), examiners reported the positivity of lesion on patients' individual records.

\subsection{Definitive diagnosis (Gold standard)}

In patients with lesions detected through any of the four examinations, it was performed incisional biopsy of the lesion. Specimens were fixed in formalin $10 \%$ and histopathologic analysis were performed, leading to the final diagnosis of each lesion.

\subsection{Data analysis}

Through descriptive analysis we performed the collection, organization and description of data obtained. In this analysis we evaluated the following variables: Sensitivity, specificity, positive predictive value (PPV), negative predictive value (NPV) and accuracy for each examination patients underwent, taking as diagnosis Gold Standard method, the histopathologic analysis.

\section{Results}

Among 15 patients included in this study, five (33.3\%) lesions with malignant risk were diagnosed, among which two (40\%) demonstrated epithelial dysplasia. One of the dysplastic lesions was not detected neither by the dentistry student nor by the adept examiner using white-light examination, and it was included in the group of lesions with malignancy risk for demonstrating dysplasia, which is an indicator of malignant transformation. Oral leukoplakia was the most common lesion detected (60\%), followed by actinic cheilitis (20\%).

\subsection{Fluorescence visualization efficacy for detecting oral lesions more prone to be dysplastic}

As seen in Table 1, unskilled examiner sensitivity, PPV, NPV and accuracy for detecting oral lesions more prone to be dysplastic among patients included in this study increased when using fluorescence visualization, although, the same did not happened with specificity value. Observing the same variables for the efficacy of adept professional in oral diagnosis in detecting oral lesions more prone to be dysplastic, it is possible to observe although sensitivity and accuracy values did not increase using fluorescence visualization, specificity, PPV, NPV did.

When comparing both examiners, it is possible to observe that when using fluorescence visualization aid, unskilled examiner's efficacy for detecting dysplasia was improved to values compatible with the adept examiner. Although specificity, PPV and NPV values for adept professional efficacy in detecting oral lesions which demonstrated to be dysplastic in histopathologic analysis increased with the aid of fluorescence visualization, this improvement for unskilled examiner were more notable (Table 1 ). 
Table 1

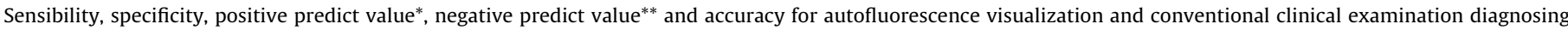
epithelial dysplasia.

\begin{tabular}{|c|c|c|c|c|}
\hline & $\begin{array}{l}\text { Unskilled examiner } \\
\text { (conventional clinical } \\
\text { examination) }\end{array}$ & $\begin{array}{l}\text { Unskilled examiner } \\
\text { (fluorescence visualization) }\end{array}$ & $\begin{array}{l}\text { Adept examiner (conventional } \\
\text { clinical examination) }\end{array}$ & $\begin{array}{l}\text { Adept examiner (fluorescence } \\
\text { visualization) }\end{array}$ \\
\hline Sensibility & $50 \%$ & $100 \%$ & $100 \%$ & $100 \%$ \\
\hline Specificity & $46.15 \%$ & $46.15 \%$ & $38 \%$ & $46 \%$ \\
\hline PPV & $12.5 \%$ & $22.22 \%$ & $20 \%$ & $22.22 \%$ \\
\hline NPV & $85.71 \%$ & $100 \%$ & $100 \%$ & $100 \%$ \\
\hline Accuracy & $46.66 \%$ & $53.33 \%$ & $46.66 \%$ & $53.33 \%$ \\
\hline
\end{tabular}

Table 2

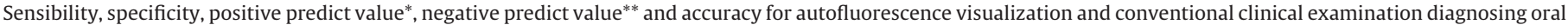
potentially malignant disorders.

\begin{tabular}{|c|c|c|c|c|}
\hline & $\begin{array}{l}\text { Unskilled examiner } \\
\text { (conventional clinical } \\
\text { examination) }\end{array}$ & $\begin{array}{l}\text { Unskilled examiner } \\
\text { (fluorescence visualization) }\end{array}$ & $\begin{array}{l}\text { Adept examiner (conventional } \\
\text { clinical examination) }\end{array}$ & $\begin{array}{l}\text { Adept examiner } \\
\text { (fluorescence visualization) }\end{array}$ \\
\hline Sensibility & $60 \%$ & $100 \%$ & $100 \%$ & $100 \%$ \\
\hline Specificity & $50 \%$ & $60 \%$ & $50 \%$ & $60 \%$ \\
\hline PPV & $37.5 \%$ & $55.55 \%$ & $50 \%$ & $55.55 \%$ \\
\hline NPV & $71.42 \%$ & $100 \%$ & $100 \%$ & $100 \%$ \\
\hline Accuracy & $53.33 \%$ & $73.33 \%$ & $66.66 \%$ & $73.33 \%$ \\
\hline
\end{tabular}

\subsection{Fluorescence visualization efficacy for detecting OPMD}

For detecting OPMD, specificity values were similar for both examiners This value was increased when oral examination was performed with the aid of fluorescence visualization. Adept professional in oral diagnosis NPV was not altered for detecting OPMD (100\%) with the aid of fluorescence visualization (Table 2 ).

Nevertheless, when using fluorescence visualization aid, unskilled examiner sensitivity, specificity, PPV, NPV and accuracy values were all improved. The same is observed for adept examiner specificity, PPV and accuracy values, nevertheless, for this examiner, increases were more discrete. It is important to notice that once enhanced with the aid of fluorescence visualization, variables values for unskilled examiner is compatible with the same values for adept examiner (Table 2).

\section{Discussion}

Fluorescence visualization had demonstrated high efficacy for detecting early oral neoplastic transformation, nevertheless, few clinical studies evaluating the efficacy of this method in general population scanning have been conducted, and in most of studies, examination by fluorescence visualization is performed by adept professional in oral diagnosis and with wide knowledge regarding the method [13].

Our results demonstrated that the use of fluorescence visualization increased sensitivity, PPV, NPV and accuracy values for the inexperienced examiner to detect oral lesions more prone to be dysplastic when compared to its conventional clinical oral examination, while specificity value was not altered. These results suggest fluorescence visualization might enhance the capacity of the inexperienced examiner not only for detecting oral lesions more prone to be dysplastic, but furthermore, for correctly provide real positive and real negative results. In 2006, when the first hand-held fluorescence visualization device was evaluated by Lane et al. [8], authors reported sensitivity and specificity values of $98 \%$ and $100 \%$, respectively, for discriminating normal epithelium from dysplasia. In study performed by Jayaprakash et al. [14], fluorescence visualization associated to white-light examination provided higher values of sensitivity than white-light examination alone for detecting high-grade lesions (from $71 \%$ to $100 \%$ ), corroborating with our results. However, in these studies, the efficacy of the method used by an unskilled professional was not assessed. Apart from that, in our study, accuracy for detecting oral lesions more prone to be dysplastic increased from $46.66 \%$ (white light examination) to $53.33 \%$ (fluorescence visualization), corroborating with results published by Farah et al. [15], in which fluorescence visualization accuracy for detecting oral lesions with dysplastic features in histopathologic analysis was $55 \%$.

Although discrete, it is also possible to observe improvements in the efficacy of both examiners (more discrete for the adept professional than for unskilled examiner) for detecting OPMD when using fluorescence visualization. However, adept professional in oral medicine sensitivity $(100 \%)$ and NPV $(100 \%)$ using whitelight examination were not altered when this professional used fluorescence visualization, which indicates this professional was capable of providing real positive results for OPMD independently of fluorescence visualization, as well as being able of accurately considering non-sick patients. Moreover, it is also possible to observe that an unskilled examiner was capable of matching its efficacy for detecting OPMD to adept professional's efficacy, when aided by fluorescence visualization. In 2013, Hanken et al. [11] also demonstrated fluorescence visualization improved sensitivity for detecting OPMD (from $75 \%$ to $97.9 \%$ ) in values equivalent to ours for an adept professional. Nevertheless, specificity values in our study were higher when both examiners used fluorescence visualization aid (from 50\% to 60\%), while Hanken et al. [11] reported specificity value for detecting OPMD increased from 33.3\% to $41 \%$ with fluorescence visualization aid. Moreover, corroborating with Farah et al. [15], in our study oral leukoplakia was the most frequent OPMD diagnosed.

The evidence found in this pilot study indicates fluorescence visualization might possibly help to improve generalist dental practitioners' potential for detecting oral risky lesions and refer these patients to specialized care for obtaining correct diagnosis and early adequate treatments. Unfortunately, there is not sufficient evidence in literature which supports the efficacy of this method in general population scanning for oral lesions with malignant risk, and that is the reason we are further conducting research with wider samples and suggesting the need for more studies regarding this issue using different methodologies aiming to clarify the real effectiveness of tissue fluorescence visualization for detecting sings of oral early neoplastic processes. It is also important to notice, fluorescence visualization might serve as an aid for evidencing oral 
lesions and should not be seen as a Golden Standard or first choice in oral diagnosis process.

\section{Conflicts of interest}

The authors declare there are no potential conflict of interest regarding this work.

\section{Acknowledgments}

The authors acknowledge the Optics and Photonics Research Center of the Physics Institute (University of São Paulo-USP, Brazil) for providing the device EVINCE ${ }^{\circledR}$ (MMOptics, São Carlos, SP, Brazil) used in this study.

\section{References}

[1] A. Jemal, F. Bray, M.M. Center, J. Ferlay, E. Ward, D. Forman, Global cancer statistics, CA: Cancer J. Clin. 61 (2011) 69-90.

[2] V. Panzarella, G. Pizzo, F. Calvino, D. Compilato, G. Colella, G. Campisi, Diagnostic delay in oral squamous cell carcinoma: the role of cognitive and psychological variables, Int. J. Oral Sci. 6 (2014) 39-45.

[3] A. Policard, A. Leulier, Caracterisation de l'haemato-porphyrine et de l'urobiline urinaire par la lumiere de wood and etude sur les aspects offerts par des tumour experimentales examinees a la lumiere de wood, CR Soc. Biol. 91 (1924) 1422.

[4] A.L.N. Francisco, W.R. Correr, L.H. Azevedo, V.G. Kern, C.A.L. Pinto, L.P. Kowalski, C. Kurachi, Fluorescence spectroscopy for the detection of potentially malignant disorders and squamous cell carcinoma of the oral cavity, Photodiagn. Photodyn. Ther. 11 (2014) 82-90.
[5] A.L.N. Francisco, W.R. Correr, C.A.L. Pinto, J. Gonçalves Filho, T.C. Chulam, C. Kurachi, L.P. Kowalski, Analysis of surgical margins in oral cancer using in situ fluorescence spectroscopy, Oral Oncol. 50 (2014) 593-599.

[6] E. Svistun, R. Alizadeh-Naderi, A. El-Naggar, A. Gillenwater, R. Richards-Kortum, Vision enhancement system for detection of oral cavity neoplasia based on autofluorescence, Head Neck 26 (2004) 205-215.

[7] J.R. Lakowicz, Principles of Fluorescence Spectroscopy, third ed., Baltimore, 2006.

[8] P.M. Lane, T. Gilhuly, P. Whitehead, H. Zeng, C.F. Poh, S. Ng, P.M. Williams, L. Zhang, M.P. Rosin, C.E. MacAulay, Simple device for the direct visualization of oral-cavity tissue fluorescence, J. Biomed. Opt. 11 (2006), 024006-024006.

[9] L.S. Junior, L.G.P. Paleckis, R.A. Nicolau, G.V. Nogueira, R.Z. Busanello, D.A. Mardegan, S.M. Fonseca, R.A. Zângaro, M.T.T. Pacheco, Detecção de lesões neoplásicas induzidas em mucosa oral de hamster utilizando espectroscopia de fluorescência, Rev. Assoc. Med. Bras. 50 (2004) 297-301.

[10] K. Huff, P.C. Stark, L.W. Solomon, Sensitivity of direct tissue fluorescence visualization in screening for oral premalignant lesions in general practice, Gen. Dent. 57 (2009) 34-38.

[11] H. Hanken, J. Kraatz, R. Smeets, M. Heiland, M. Blessmann, W. Eichhorn, T.S. Clauditz, A. Gröbe, A. Kolk, M. Rana, The detection of oral pre-malignant lesions with an autofluorescence based imaging system (VELscope TM)-a single blinded clinical evaluation, Head Face Med. 9 (2013) 1.

[12] L. Sweeny, N.R. Dean, J.S. Magnuson, W.R. Carroll, L. Clemons, E.L. Rosenthal, Assessment of tissue autofluorescence and reflectance for oral cavity cancer screening, Otolaryngol. Head Neck Surg. 145 (2011) 956-960.

[13] D.M. Laronde, P.M. Williams, T.G. Hislop, C. Poh, S. Ng, C. Bajdik, et al., Influence of fluorescence on screening decisions for oral mucosal lesions in community dental practices, J. Oral Pathol. Med. 43 (2014) 7-13.

[14] V. Jayaprakash, M. Sullivan, M. Merzianu, N. Rigual, T.R. Loree, S.R. Popat, et al., Autofluorescence-guided surveillance for oral cancer, Cancer Prev. Res. 11 (2009) 966-974.

[15] C.S. Farah, L. McIntosh, A. Georgiou, M.J. McCullough, Efficacy of tissue autofluorescence imaging (VELScope) in the visualization of oral mucosal lesions, Head Neck 34 (2012) 856-862. 